\title{
EDITORIAL - SOZIALISATIONSFORSCHUNG REVISITED
}

\section{Fränzi Buser \& Peter Rieker}

Was verbindet Gesellschaft und Individuum? Wie prägen gesellschaftliche Ordnungen Individuen? Wie werden gesellschaftliche Institutionen von Individuen im Zusammenspiel mit anderen Individuen hervorgebracht und dabei mitunter auch verändert? Fragen wie diese in Bezug auf die Vermittlung von Gesellschaft und Individuum beschäftigen die Sozialisationsforschung schon seit geraumer Zeit, sie werden vielfältig und zuweilen kontrovers bearbeitet und diskutiert. Ausgehend von klassischen, strukturfunktionalistischen Sozialisationstheorien sowie darauf bezogener Kritik, etwa von Seiten interaktionistischer Theoretiker*innen, haben sich die Debatten in der Sozialisationsforschung stetig weiterverändert und gewandelt, sodass gegenwärtig von einem breiten, facettenreichen Theorie- und Forschungsfeld gesprochen werden kann. Die verschiedenen Felder, Instanzen und thematischen Aspekte von Sozialisation werden dabei auch aus interdisziplinärer Perspektive beleuchtet, wobei etwa Bedingungen, Verläufe, Ausprägungen oder Ergebnisse von Sozialisationsprozessen in den Blick genommen werden. Gegenwärtig scheinen beispielsweise das Verständnis von Sozialisation als wechselseitiger und lebenslanger Prozess oder Konzepte von individueller Entwicklung im Sinne eines Selbstsozialisationsprozesses im Trend zu liegen.

Ausgehend vom Eindruck, dass Sozialisationsforschung in der Zeitschriftenlandschaft bisher eher untervertreten ist, haben wir uns entschieden eine Zeitschrift zu gründen, welche als Forum für die vielfältigen Fragen und Debatten aus diesem breiten Forschungsfeld dienen kann. Mit „Gesellschaft - Individuum - Sozialisation" (GISo) erscheint nun eine neue Zeitschrift für Sozialisationsforschung, die das bestehende Spektrum von Fachzeitschriften ergänzen soll. GISo bietet eine Plattform für die Publikation wissenschaftlicher Originalbeiträge aus den Sozialwissenschaften, der Psychologie und der
Geschichtswissenschaft sowie den Erziehungsbzw. Bildungswissenschaften. Ziel ist es, ein unabhängiges und interdisziplinäres Forum für die Präsentation und Diskussion theoretischer und konzeptioneller Positionen, empirischer Ergebnisse und forschungsmethodischer Herausforderungen im Feld der Sozialisationsforschung anzubieten. Dabei ist die Zeitschrift offen für Fragen der Sozialisation in verschiedenen Lebensphasen und Kontexten, in denen jeweils spezifische soziale Beziehungen, Prozesse und Bedingungen einzubeziehen und gesellschaftliche Dimensionen (z.B. Geschlecht, Migration, sozioökonomische Dominanz- und Ungleichheitsverhältnisse) zu berücksichtigen sind. Vor diesem Hintergrund können Prozesse in informellen Sozialisations- und Erziehungskontexten, aber auch institutionelle Bildungs- und Betreuungsangebote sowie gesellschaftliche, politische und kulturelle Prozesse in den Blick genommen werden.

In der vorliegenden ersten GISo-Ausgabe "Sozialisationsforschung revisited" finden sich Beiträge aus unterschiedlichen Bereichen der Sozialisationsforschung, wobei vor allem innovative Potenziale in diesem Forschungsfeld aufgezeigt werden. Skizziert werden theoretische Konzeptionen, welche Sozialisation weiterdenken, neue Überlegungen zu den Grundfragen der Sozialisationsforschung und empirische Arbeiten zu bisher wenig etablierten Feldern dieses Forschungsbereiches. Auf diese Weise werden nicht nur die Potenziale der Sozialisationsforschung deutlich, sondern es zeigen sich auch Anschlüsse zu verschiedenen Diskussionen und Forschungsfeldern sowie Möglichkeiten, diese weiter zu entwickeln. Die im ersten Heft versammelten Beiträge blicken hierbei mit unterschiedlichen theoretischen Brillen und mittels verschiedener empirischer Zugänge auf Fragen der Sozialisation. 
Mechthild Bereswill und Gudrun Ehlert setzen sich im ersten Beitrag vertieft mit strittigen Positionen zu Sozialisation und Geschlecht auseinander. Dabei problematisieren sie am Beispiel von Geschlecht die Integration von biologischer und sozialwissenschaftlicher Forschung. Ausgehend von der Kritik an sozial- und kulturwissenschaftlichen Ansätzen der Geschlechterforschung, die „biologische Natur" des Menschen nicht zu berücksichtigen, setzen sie sich mit Befunden der sozialwissenschaftlichen Geschlechterforschung auseinander und nehmen auf ausgewählte Befunde und Diskussionen der Neurowissenschaften sowie der Evolutionspsychologie Bezug. Die Autorinnen kommen zum Schluss, dass die Sozialisation von Menschen nicht als "Disposition plus Umwelt" gedacht werden könne, sondern vielmehr als komplexe, historische und wandelbare Sozialität in den Blick genommen werden müsste. Insofern plädieren sie für eine Weiterentwicklung und Stärkung der sozialwissenschaftlichen Geschlechterforschung zu Sozialisation.

Im darauffolgenden Beitrag stellt Alexandra König auf der Basis einer qualitativen Längsschnittstudie zu Ausbildung und Studium das heuristische Modell ,.,Sozialisation als Selbstprojekt" vor, welches Sozialisation aus interaktionistischer und ungleichheitsanalytischer Perspektive gleichermassen zu fassen sucht. Am Beispiel der Ausbildung in kleinen Handwerksbetrieben sowie des Lehramts- und Ingenieursstudiums an Hochschulen erörtert sie im Dialog zwischen theoretischen Vorannahmen und empirischem Material das Selbstprojekt als Schlüsselkonzept, das drei Ebenen bündelt: Sozialisation innerhalb eines spezifisch strukturierten Feldes, Sozialisation als interaktives Geschehen und Sozialisation als Prozess der Formung des Selbst. Dies erlaubt es, so die Autorin, Studierende und Auszubildende aus einer gemeinsamen sozialisationstheoretischen Perspektive zu betrachten und Ungleichheiten der Statuspassage sowie Ungleichheiten, die durch die Feldspezifik der Statuspassage evoziert werden, zu untersuchen.

Peter Rieker widmet sich in seinem Beitrag einem bisher vernachlässigten Forschungsfeld innerhalb der Sozialisationsforschung, indem er Aushandlungs- und Entscheidungsprozesse in
Peergroups Jugendlicher fokussiert. Ausgehend von ethnographischen Beobachtungen aus drei verschiedenen Peerkontexten rekonstruiert er kontextspezifische Ausprägungen von Entscheidungsprozessen sowie verschiedene Varianten von Aushandlungen, die im Hinblick auf Entscheidungen festzustellen sind. Zudem arbeitet er unterschiedliche Konstellationen von Entscheidungsprozessen heraus und interpretiert diese im längsschnittlichen Zusammenhang. Schliesslich diskutiert er auch die im Peerkontext festzustellenden Ungleichheiten in ihrer Relevanz für Entscheidungsprozesse. Gemäss dem Autor bestätigen die Ergebnisse die Position interaktionistischer Sozialisationstheorien und unterstreichen den Aushandlungscharakter sowie die Variabilität peerspezifischer Sozialisationsprozesse.

Konfessionelle Heimerziehung als ein weiteres bisher in der Sozialisationsforschung wenig berücksichtigtes Feld steht im darauffolgenden Beitrag von Daniela Reimer im Fokus. Die Autorin stellt hierbei eine explorative Studie zu christlichreligiösen Praktiken im Alltag der Heimgruppe eines katholischen Trägers in Deutschland vor, wobei sie mittels teilnehmender Beobachtungen und halboffener Interviews mit verschiedenen Zielgruppen religiöse Praktiken im Alltag der konfessionellen Heimerziehung und ihre Legitimationen durch Leitungs- und Fachkräfte untersucht. Dabei zeigt die Autorin auf, dass religiöse Praktiken den Alltag in der Heimgruppe durchziehen, ohne dass dies den Fachkräften bewusst zu sein scheint und dass sie selbstverständlich und unhinterfragt praktiziert und mitunter normierend und moralisierend eingesetzt werden. Fachkräfte legitimieren religiöse Praktiken insbesondere mit dem christlichen Träger, mit persönlichen Überzeugungen sowie dem resilienzfördernden Potenzial, welches religiösen Praktiken generalisierend zugeschrieben wird. Bilanzierend verweist die Autorin auf Themen, welche es in der weiteren Forschung in diesem Feld zu vertiefen gelte und plädiertfür einen Fachdiskurs innerhalb der Sozialpädagogik, in dem die religiöse Dimension nicht länger de-thematisiert werden sollte.

Gudrun Quenzel und Katharina Meusburger fokussieren in ihrem Beitrag auf das Erklärungspotenzial von Schule, Eltern, Freund*innen und 
Freizeit für das Auftreten von psycho-somatischen Beschwerden von Mädchen und Jungen. Ausgehend von einer repräsentativen Befragung von Schüler*innen in Vorarlberg, Österreich, fragen sie nach möglichen Unterschieden in den Bedingungen des Aufwachsens von Mädchen und Jungen als Erklärung dafür, weshalb psychosomatische Beschwerden bei Mädchen stärker auftreten als bei Jungen. Dabei zeigen sie mittels linearer Regressionsanalysen, dass die Unzufriedenheit mit den eigenen Schulleistungen den grössten Einfluss auf das Auftreten von psychosomatischen Beschwerden hat, gefolgt von negativen Körperwahrnehmungen. Der Beitrag verweist auf die Relevanz des Sozialisationskontextes für das Verständnis von Gesundheit im Jugendalter und bestätigt, dass die geschlechtliche Zugehörigkeit mit den sozialen Faktoren interagiert, welche die Gesundheit beeinflussen.

Ergänzt werden diese Beiträge durch eine Rezension von Rebecca Mörgen zu dem von Daniela Reimer herausgegebenen Sammelband „Sozialpädagogische Blicke“. Dieser wurde Klaus
Wolf anlässlich seiner Emeritierung gewidmet und lädt die Leser*innen - so die Rezensentin ein, sozialpädagogische Prozesse, die institutionelle Bearbeitung von subjektiven Lebensführungsweisen und etablierte Konzepte innerhalb der Sozialpädagogik als Disziplin und Profession zu reflektieren. Abschliessend blickt Selin Kilic in ihrem Bericht auf die internationale Tagung „Migration. Erinnern. Praktiken des Erzählens und Erinnerns in der Migrationsgesellschaft" zurück, welche im Herbst 2019 an der Universität Zürich stattfand. Dabei lässt sie die einzelnen Beiträge sowie ein Forschungsgespräch, das die Vorträge ergänzte, Revue passieren und bilanziert den Ertrag der Tagung.

Wir hoffen, mit dieser Ausgabe interessante Einblicke in aktuelle Forschungsarbeiten und Debatten der Sozialisationsforschung zu geben. Wir wünschen allen Leser*innen eine anregende und spannende Lektüre und laden dazu ein, das Feld der Sozialisationsforschung in seiner Vielfältigkeit in den Blick zu nehmen und hierbei Sozialisation auch weiter und neu zu denken!

\section{Die Redaktion}

Fränzi Buser ist wissenschaftliche Assistentin am Lehrstuhl für Ausserschulische Bildung und Erziehung am Institut für Erziehungswissenschaft der Universität Zürich. Sie forscht zu Kindheit, Migration und Flucht, Intersektionalität, Bildung und sozialer Ungleichheit.

Peter Rieker leitet den Lehrstuhl für Ausserschulische Bildung und Erziehung am Institut für Erziehungswissenschaft der Universität Zürich. Er forscht zu Kindheit und Jugend, zu abweichendem Verhalten und Extremismus sowie zu politischer Partizipation, Migration und interethnischen Beziehungen. Zudem arbeitet er zu Methoden der qualitativen Sozialforschung und zu multimethodischer Forschung.

Kontakt

Fränzi Buser

Prof. Dr. Peter Rieker

Institut für Erziehungswissenschaft

Universität Zürich

Freiestr. 36

$\mathrm{CH}-8032$ Zürich

Tel.: +414463427 79

E-Mail: fraenzi.buser@ife.uzh.ch

URL: https://www.ife.uzh.ch/de/research/abe/mitarbeitende2/buserfranziska.html

Tel.: +41446344561

E-Mail:prieker@ife.uzh.ch

URL: https://www.ife.uzh.ch/de/research/abe/mitarbeitende2/riekerpeter.html 
(C) 